\title{
Weighting Parties and Coalitions: How Coalition Signals Influence Voting Behavior
}

\author{
Thomas Gschwend, University of Mannheim \\ Michael F. Meffert, Leiden University \\ Lukas F. Stoetzer, Massachusetts Institute of Technology
}

Democratic accountability is characterized as weak in parliamentary systems where voters cannot choose their government directly. We argue that coalition signals about desirable and undesirable coalitions that might be formed after the election help to provide this essential aspect of democratic government. We propose a simple model that identifies the effect of coalition signals on individual vote decisions. Based on survey experiments in two different countries we show how coalition signals change the relative weight of voters' party and coalition considerations. Coalition signals increase the importance of coalition considerations and, at the same time, decrease the importance of party considerations in voters' decision calculus, leading some voters to change their vote intention.

A central feature of democratic representation is that the government actively works to foster congruence between the preferences of the electorate and the government even if the preferences of the electorate change. The mechanisms to secure that and to hold the government accountable are popular elections. As long as there are singleparty governments, this is in fact a straightforward process. However, in most democratic parliaments we do not find a single party that has a legislative majority of seats. Rather, coalition governments have to be formed after the election (Hobolt and Karp 2010; Kedar 2011; Meffert and Gschwend 2010). Does this necessarily imply that democratic accountability is seriously hampered? In contrast to the textbook view on democratic institutions we argue that coalition signalsa not well-understood feature of political campaigns - can foster accountability at least as a by-product even in situations where voters cannot choose their government directly.

During election campaigns parties often announce which coalition they might or might not form after the election depending on the outcome on election day. Such "coalition signals" are prominently covered by the media, which is then eager to further speculate about the composition of the next government. In practice, coalition signals can offer crucial information to voters (Gschwend, Stoetzer, and Zittlau 2016). As one preelectoral coalition strategy (Golder 2005, 2006), coalition signals may provide guidance about which coalition governments are conceivable politically and likely to gain a majority in parliament. They reduce the number of theoretically possible coalitions to a manageable range and help citizens to form clearer expectations about the government formation process after the election. A coalition of parties, for instance, that signaled their intention to govern together before the election can reasonably claim to have won a mandate after the election if they managed to get a majority of seats in parliament.

Arguably, preelectoral coalitions have important implications for the nature of representative governments. If voters have clearer expectations about government formation after the election, they are better able to assess the potential (policy) consequences of voting particular coalitions into office. This way coalition signals facilitate clarity of responsibility, accountability, and identifiability of future governments even in

\footnotetext{
Thomas Gschwend (gschwend@uni-mannheim.de) is a professor at Mannheim University, Department of Political Science, and Mannheim Centre for European Social Research (MZES), 68131 Mannheim, Germany. Michael F. Meffert (m.f.meffert@fsw.leidenuniv.nl) is a lecturer at the Institute of Political Science at Leiden University, 2333 Leiden, Netherlands. Lukas F. Stoetzer (lstoetze@mit.edu) is a postdoctoral fellow at the Massachusetts Institute of Technology, Department of Political Science, Cambridge, MA 02139.

Data and supporting materials necessary to reproduce the numerical results in the article are available in the JOP Dataverse (https://dataverse.harvard .edu/dataverse/jop). An online appendix with supplementary material is available at http://dx.doi.org/10.1086/688678.
}

The Journal of Politics, volume 79, number 2. Published online January 24, 2017. http://dx.doi.org/10.1086/688678

(C) 2017 by the Southern Political Science Association. All rights reserved. 0022-3816/2017/7902-0019\$10.00 
systems that do not per se employ majoritarian institutions. This useful function for promoting these essential aspects of democratic government has long been noted in the literature (Clark, Golder, and Golder 2012; Powell 2000; Strom 1990).

Given their prominence in everyday politics as well as their theoretical and practical importance for "good" voting decisions in complex multiparty environments, evidence about how coalition signals work at the individual level is mostly and surprisingly lacking. In this article we present a simple model that allows to directly estimate the effect of coalition signals on individual voting decisions. By using unique survey experiments in two different countries, we find that coalition signals have an effect on voters' decision calculus by changing the relative weights in voters' utility function. Coalition signals increase the importance of coalition considerations and, at the same time, decrease the importance of party considerations. By developing a novel methodological approach to estimate decision weights of different but competing considerations, we give coalition signals and their potential effects on vote decisions the attention they deserve.

Placing more weight on coalition preferences relative to party preferences when deciding which party to select matters for accountability because coalitions (and not merely a party) can be held accountable for government policy. If coalitions are identifiable, the process to anticipate who will be part of the new government is more transparent and predictable (Debus 2009; Martin and Stevenson 2001, 2010). Thus, voters are enabled to register their support in such a way that an incumbent coalition government perceived as incompetent is voted out of office and replaced by a new coalition government that promises to better represent their policy preferences.

\section{WHY COALITION SIGNALS MATTER: THE EXISTING EVIDENCE}

Coalition governments are very common in parliamentary democracies, creating an electoral environment and incentives that differ considerably from the familiar two-party competition in textbook examples. For parties, one preelectoral strategy is to send out coalition signals during campaigns. Such signals can be defined as any official or unofficial preelectoral statement by a party, party members, or other political actors about coalitions that might or might not be formed after the next election.

Empirically, preelectoral coalitions and preelectoral coordination are fairly common among parties during campaigns (Golder 2005, 2006). Very common are joint campaign events or press conference in which the respective party leaders appear side by side. For example, during the 1998 German election, the leaders of the Social Democrats (Gerhard Schröder,
SPD) and the Green Party (Joschka Fischer, Greens) who eventually succeeded the Helmut Kohl-led Christian-liberal (CDU-FDP) government, campaigned together. In many Scandinavian countries party leaders of the alternative coalition to the incumbent government organize common press conferences (Fredén 2014). Sometimes, such as in Norway (2005), the coalition parties even develop common platforms before the election (Allern and Aylott 2009) or coordinate their candidate entries such as in Portugal (Gschwend 2007a) or France (Blais and Indridason 2008). Viewers of the fictional Danish TV series "Borgen" - a (functional) equivalent of "House of Cards"- will be familiar with vivid illustrations of such coordination.

For a voter, multiparty systems might appear to make it extremely difficult to anticipate the future government and then to vote for a coalition party that will move the new government's policy output as closely as possible to the voter's position. It requires that this party is not only represented in parliament (e.g., by passing some minimum vote threshold) but also becomes a member of the next coalition. However, this might be easier than it first appears. As "members of the polity" (Lewis-Beck and Skalaban 1989), citizens tend to be familiar with the political history and the political parties of a country (Armstrong and Duch 2010; Gschwend 2007b; Herrmann 2014). Parties are real and existing organizations and thus well-known to voters. Coalitions, on the other hand, are mostly hypothetical constructs with the notable exception of the current incumbent coalition and any other recent coalition government. In short, voters possess a basic political coordinate system that helps them to rule out implausible coalitions among the many theoretically possible ones. Nevertheless, in order to predict viable coalition alternatives prior to an election, citizens need more current and pertinent information.

Besides preelection polls, coalition signals are the most obvious source of such information. Parties might use such signals to announce a preferred coalition partner or rule out other parties as unacceptable in a future coalition government. If voters take such signals into account, they might adjust and change their vote intentions based on the expected outcome of the election. And history shows, those signals are not just cheap talk for voters. They have real consequences. Parties get punished if they do not keep them. In the $1996 \mathrm{New}$ Zealand election, for instance, "New Zealand First" clearly signaled that it would not form a coalition government with "National" but then did not keep this promise (Bowler, Karp, and Donovan 2010). As a consequence, the party lost about two-thirds of its support in the next election.

How do voters take coalition signals into account? Take, for example, an instrumental voter who prefers a party that 
is unlikely to join the next government. If other parties signal that they like to form the next government, this voter could defect from her preferred party and rather cast a vote for the most preferred party among the expected coalition parties in order to influence the composition and portfolio of the next government. There is evidence from recent Israeli and Austrian election that, in particular, small party supporters systematically apply this type of coalition voting strategy (Bargsted and Kedar 2009; Herrmann 2014; Meffert and Gschwend 2010).

Coalition signals could also prime voters to support another party in order to prevent a likely coalition. If a voter dislikes the signaled coalition partner of her most preferred party, she might defect from her preferred party and instead cast her vote for a party that makes this coalition less likely. Evidence from recent Austrian, German, and Belgian elections is consistent with this type of voting behavior in multiparty systems (Gschwend and Hooghe 2008; Herrmann 2014; Linhart 2009; Meffert et al. 2011). In short, the recent literature indicates that coalition considerations matter above and beyond party considerations for electoral behavior in multiparty systems (e.g., Aldrich et al. 2004; Bargsted and Kedar 2009; Blais et al. 2006; Duch, May, and Armstrong 2010; Kedar 2011; Meffert and Gschwend 2010). What is less clear, however, is how to identify and estimate the effects of these different considerations. In a given electoral contest, the key causal factors - coalition signals and party preferences - do not vary much, making causal inferences very difficult.

One solution is to conduct counterfactual simulations. Linhart (2009), for example, starts with a formal model of a voter's decision-making process in a multiparty system where voters anticipate the coalition bargaining process and the resulting position of the new government using coalition signals. Combining measures of observed coalition signals before the 2005 German Federal election with various counterfactual simulations, he demonstrates that coalition considerations (should) have a strong impact on instrumental voters. Incidentally, he also identifies conditions under which coalition signals are an efficient tool in a party's electoral strategy.

More direct evidence comes from laboratory experiments that allow the creation of theoretically relevant decision scenarios by manipulating coalition signals and testing their effect on voters. Coalition signals have been used as a treatment in an economic experiment to test a decision-theoretic model of vote choice in a multiparty system (Meffert and Gschwend 2012). Participants with an instrumental (financial) motivation were asked to vote in various elections in which the availability of polls and coalition signals was manipulated to test under what conditions strategic voting happens. The results show that participants rely on simple decision heuristics in order to behave strategically and are highly susceptible to coalition signals. ${ }^{1}$

A weakness of such laboratory experiments is external validity, making randomized experiments embedded in representative surveys an ideal compromise to address the effect of coalition signals. Irwin and Van Holsteyn (2012), for example, operationalized coalition signals as a part of vignettes in a survey experiment in the Netherlands (see also Irwin and Van Holsteyn 2008). These vignettes presented respondents with hypothetical but plausible results of opinion polls and their consequences for the formation of the next coalition government. The results suggest that some voters even used these vignettes to actually develop an initial vote intention, while those with a vote intention, in particular supporters of small parties, sometimes changed their vote intention. If the preferred small party was a member of the next coalition (according to the vignette), voters were more likely to support the preferred party. But if the small party was not in the coalition, supporters of small parties were more likely to defect (compared to supporters of large parties). This suggests that small party supporters are more likely to engage in strategic coalition voting behavior. Nevertheless, vignettes that combine poll results with coalition signals cannot isolate the effect of coalition signals from the effects of poll results, leaving the question of distinct coalition signal effects open.

In summary, the limited existing evidence suggests that coalition signals can be quite influential and are used by voters to adjust their vote intentions. In the next sections, we propose a simple mechanism that can explain such effects - coalition signals prime coalition considerations at the expense of party considerations - as well as a statistical model for survey experiments that can estimate the shifting weights of the different considerations. Based on this model, it is possible to identify which coalition signals lead to changes in vote decisions.

\section{HOW COALITION SIGNALS MATTER: A THEORETICAL DECISION MODEL}

The previous section suggests that coalition signals matter. However, the current literature is rather agnostic about the processes by which they should exert an influence on an individual's decision to vote. In this section we propose a model that reflects the systematic component of such a decision-making process. We then derive a particular observable implication of this model that can be tested with a survey experiment.

1. In a psychological experiment embedded in two actual state election campaigns in Germany, Meffert and Gschwend (2011) tested the effects of coalition signals on voting behavior for real parties. The results again suggest that coalition signals did increase the likelihood of defection from the preferred party. 
Our model starts with the premise that voters are able to form considerations about parties as well as coalitions. The established theory of political behavior building on insights derived from case-studies about the United States suggests, of course, that party considerations should play a dominant role in the way people make up their mind. Parties are "real," after all. Thus, one constituent part in a voter's utility function should depend on a party-centered component, how much she prefers different parties. In order to focus our discussion, we leave aside questions to what degree those party preferences are rooted in valence assessments, ideological judgments, or something else.

Given the state of the literature about voting behavior in multiparty settings, though, it is by now fairly uncontroversial to assume that voters also use a second, coalition-centered component in their vote-choice function. ${ }^{2}$ Again, the focus is not on the origin of such coalition preferences. In essence, we assume merely that coalition considerations could be one relevant additional yardstick for voters. Given that typically no single party wins an outright majority of seats in parliament, voters are motivated to support the coalition they prefer most or to increase the likelihood that a coalition government perceived as incompetent is voted out of office. This way voters can hold coalition governments accountable.

Finally, we assume that voters can consider both components, party as well as coalition considerations, together in their decision-making process. Given that voters have already formed preexisting political attitudes about parties and (some) coalitions, it is likely that those attitudes affect the voting decision one way or another. This perspective reflects the current consensus in the literature on coalition voting (e.g., Aldrich et al. 2004; Bargsted and Kedar 2009; Blais et al. 2006; Duch et al. 2010; Gschwend et al. 2016; Kedar 2011; Meffert and Gschwend 2010). As long as party and coalition preferences are together on the right-hand side of any votechoice model, this implies that a voter-no matter how the respective systematic component of the model does parameterize her decision-making process - will employ both components simultaneously.

How do coalition signals come in? Our central argument is that coalition signals can prime coalition considerations. They greatly reduce the number of theoretically possible coalitions and help citizens to form clearer expectations about the government formation process after the election. Most importantly, they remind voters of the electoral relevance of

2. In addition to more short-term considerations about parties and coalitions there are, of course, other long-term factors such as party identification and other socio-demographic characteristics that anchor voters' decisionmaking process. preexisting political attitudes about coalitions and make these considerations more accessible to them when forming and expressing a vote intention. Once those coalition considerations are made more accessible, voters should take them (more) into account. In other words, a coalition signal about a concrete coalition should increase the importance voters attach to coalition considerations regarding this coalition relative to party considerations in their decision making process. Similar to Bartels $(2006,82)$, we use the term "priming" to refer to any systematic change in the relative weights attached to coalition and party considerations, that is, the preexisting political attitudes toward coalitions and parties that determine the vote intention, for example expressed in the context of a survey experiment.

To formalize those ideas, we define that the systematic part of the utility that voter $i$ derives from party $j$ is a mixture of the party-centered component $P_{i j}$ and a coalition-centered component $C_{i}$. Our parameter of interest, $\gamma$, is the mixing component between the two:

$$
V_{i j}=\gamma P_{i j}+(1-\gamma) C_{i}
$$

Equation (1) depicts each voter's utility as a weighted average of coalition and party considerations, with the relative weights depending on the importance of coalition considerations for her utility. Note that if $\gamma$ is equal to 1 , the model reduces to the traditional party-centered component. If $\gamma$ is equal to 0 , our model reduces to the coalition-centered component in which parties would no longer be the dominant judgmental objects for political decision making.

Of theoretical interest here is the mixing parameter $\gamma$ : To what extend do voters employ coalition considerations relative to party considerations in their vote decision? If our priming story is correct, we expect coalition signals to systematically increase the relative importance of coalition versus party considerations in an individual's decision-making process. Given our utility model from above, the observable implication would be that coalition signals should decrease the size of the mixing parameter $\gamma$. If no coalition signal is present party consideration as opposed to coalition consideration should dominate the decision-making process. A high estimated value for the respective mixing parameter $\gamma$ would support this logic. The situation should systematically change if the voter is primed with a coalition signal. If a coalition signal is present, coalition considerations regarding this coalition should become more important and, consequently, the size of the mixing parameter $\gamma$ should decrease. ${ }^{3}$

3. Alternatively, the decision-making process could be conceptualized in such a way that an individual voter uses either party or coalition considerations but not both simultaneously. Such an alternative utility 
The next section outlines our empirical strategy to test whether the mixing parameter $\gamma$ changes systematically when comparing a standard vote choice situation with one in which the voter is primed by a coalition signal. This test draws on two survey experiments, one included in an Austrian preelection study in 2006 and the other in the 2009 German Longitudinal Election Study (GLES).

\section{EMPIRICAL STRATEGY: COALITION VIGNETTES}

How can we know whether coalition signals increase voters' reliance on coalition considerations? Our empirical strategy is to embed coalition signals as vignettes in a representative population survey (for a similar strategy, see Irwin and Van Holsteyn [2008, 2012]). In fact, we leverage data from two different survey experiments in Austria and Germany to test our expectations. The presentation of a particular coalition vignette that stresses a specific coalition scenario mimics a particular real-world coalition signal and is expected to prime coalition considerations. In order to identify the effect of such a coalition signal, our general strategy across both data sets is to compare respondents' answer to the standard vote intention question, called the "standard decision" below, with responses to a second vote intention question that is asked immediately after the presentation of each coalition vignette. We call the second decision in both experiments the "vignette decision." The variance in terms of coalition considerations between the standard decision where no coalition signal is presented and each vignette decision that should prime particular coalition considerations allows us to identify the causal effect of each coalition signal. If a respondent's intention to vote changes between the two decisions, this can be directly attributed to the perception of a particular coalition vignette that presents a hypothetical coalition scenario. The implementation of the vignettes as coalition signals differs across the two studies in important aspects. Thus, we will discuss them separately.

could be written as $V_{i j}=\gamma_{i} P_{i j}+\left(1-\gamma_{i}\right) C_{i}$, where $\gamma_{i}$ is now specific for individual voters and assumed to be drawn from a Bernoulli distribution, $\gamma_{i} \sim \operatorname{Ber}(\pi)$, with a hyper-parameter $\pi$. The latter can be interpreted as the share of party-type voters in the electorate. In this alternative model coalition signals do not affect each individual's weight that reflects the importance of coalition considerations relative to party considerations but simply let more voters employ coalition considerations instead of party considerations. The impact of coalition signals is thus on the hyper-parameter $\pi$ rather than on the weight between party and coalition considerations. However, the estimated coefficients of the systematic component of such an alternative process would, on average, correspond to the mixing parameter $\gamma$ between party and coalition preferences in our model. As a consequence, we cannot empirically distinguish both processes. Further research could devise an experimental design that allows one to tease out the differences between both processes.
The first survey experiment was implemented in a preelection survey of the Austrian General Election 2006. Participants were exposed to four different vignettes. The coalition scenarios in those vignettes used coalitions that voters would accept as plausible; the two major parties, the Austrian People's Party (ÖVP) and the Social Democrats (SPÖ), were each combined with one of the smaller parties, the Greens and the Freedom Party (FPÖ). The vignettes were presented shortly after asking the standard vote intention question. They were introduced by the statement that "most parties have not made a clear announcement about possible coalitions after the election" and followed by four vignettes, in randomized order:

"For which party would you vote if the Greens would clearly reject a coalition with the SPÖ and announce the intention to form a coalition with the ÖVP?"

"For which party would you vote if the Greens would clearly reject a coalition with the ÖVP and announce the intention to form a coalition with the SPÖ?"

"For which party would you vote if the FPÖ would drop its intention to not participate in any coalition and rather announce the intention to form a coalition with the ÖVP?"

"For which party would you vote if the FPÖ would drop its intention to not participate in any coalition and rather announce the intention to form a coalition with the SPÖ?"

A different set of vignette decisions is implemented in the German Longitudinal Election Study (GLES Online Tracking T4). In this study participants were exposed to four coalition scenarios: a coalition between the Christian Democrats (CDU/ CSU) and the Greens (Bündnis 90/Die Grünen); a coalition between the Social Democrats (SPD), the Liberals (FDP), and the Green party, which is commonly referred to as the "trafficlight coalition"; a "Left Alliance" coalition between the SPD, Greens, and the Left party (Die Linke), and, finally, a socialliberal coalition between the SPD and the FDP. The wording of the question introduced these four possibilities with "Before elections, parties make statements about potential parties they want to form a coalition with. In the following we present four examples of such statements." This is followed by four vignettes starting with: "For which party would you vote if ..."

"the Greens (Bündnis 90/Die Grünen) announce the intention to join a coalition led by CDU/CSU?"

"SPD and Greens announce the intention to form a coalition with the FDP?"

"SPD and Greens announce the intention to form a coalition with the Left Party (Die Linke)?" 
"the FDP announces the intention to join a SPDled coalition?"

To sum up, both the GLES and the Austrian study include four different coalition vignettes that allow us to estimate the effect of coalition signals on vote intentions. Apart from standard party-centered measurements, such as party like/dislike scores, both studies further include equivalent measures to rate coalitions on the very same 11-point scale. We use those measures to operationalize the party-specific $\left(P_{i j}\right)$ as well as the coalition-specific $\left(C_{i}\right)$ components of equation (1) directly. ${ }^{4}$ In the next section we lay out how we are going to model a voter's decision-making process across both decisions, the standard and the vignette decision. In order to be as close as possible to our theoretical model, we will introduce a novel choice model that is tailored to directly estimate the parameter of interest $(\gamma)$.

\section{STATISTICAL MODEL TO ESTIMATE THE EFFECT OF COALITION VIGNETTES}

This section outlines a new statistical model that permits us to estimate the effect of coalition signals on respondents' reliance on coalition relative to party considerations. Given our empirical strategy we are interested in comparing the standard decision with the vignette decision to determine whether priming a voter's coalition considerations leads respondents to rely more on coalition considerations and less on party considerations. Consequently, we need to model the transition probabilities between the two decisions. If coalition signals cause voters to rely more on coalition considerations relative to party considerations, the mixing parameter $\gamma_{2}$ of the vignette decision should systematically decrease as compared to $\gamma_{1}$, the mixing parameter of the standard decision. In the following discussion we focus on coalition signals with coalitions of two parties. ${ }^{5}$

We conceptualize voters' relevant choice situation as either intending to vote for one of the two parties $\left(y_{t}=1, y_{t}=2\right)$ mentioned in the respective coalition vignette, or intending to do something else $\left(y_{t}=3\right) .^{6}$ Thus, each decision com-

4. Table 1 and table 9 in the appendix show the descriptive statistics of those variables.

5. The German data include two vignettes that prime three-party coalitions. In order to use those vignettes, we also extend the model outlined here to be applied to a situation with three parties.

6. The third category includes decisions such as "intending to vote" for any other party on the ballot or "not voting" at all. We combine these decisions as we are mostly interested in observing any reaction that pushes or pulls voters toward or away from the two parties included in the vignette. Taking all possible transitions into account is not feasible and cannot be estimated (e.g., with five parties, abstention, and a don't know/undecided category, these seven choice options already produce 49 possible transitions). prises a choice set of three different choices $j \in\{1,2,3\}$. Given that we model two sequential decisions simultaneously, $j_{1}$ represents the vote choice in the standard decision asked first, while $j_{2}$ represents the vote choice of the vignette decision that is asked second. Across both decisions we distinguish nine different outcomes $Y_{j_{1} j_{2}}{ }^{7}$ Such a sequential choice situation constitutes a first-order Markov chain process (Diggle et al. 2002; Hillygus 2007). We simultaneously estimate nine so-called transition probabilities, denoted by $\pi_{j_{1} j_{2}}$ with $\sum \pi_{j_{1} j_{2}}=1$, to represent voters' probability to change their vote choice across both decisions. ${ }^{8}$ Table 1 schematically depicts all nine transition probabilities.

In order to specify a model for those nine transition probabilities, we start by mapping out this sequential choice process, as visualized in a decision tree in figure 1. In the standard decision a respondent intends to vote for one of the parties mentioned in the coalition vignette or any third alternative. The same is true for the vignette decision. Respondent $i$ derives utility $U_{i j}^{1}$ from each of the choices in the standard decision. The utility resulting from the vignette decision can be thought of being dependent upon the standard decision. Hence, two voters who intend to vote for the same party in the vignette decision will nevertheless receive different utilities from that decision if one of them intended to vote for party A previously, while the other intended to vote for party $B$. We take this into account by conceptualizing the utility of the second vignette decision as dependent on the outcome of the first standard decision $\left(U_{i j}^{2} \mid y_{1}\right)$.

Now we can derive a statistical model that is tailored to the sequential choice processes we are interested in. ${ }^{9}$ Following random-utility theory we assume that someone who votes for the first party twice $\left(Y_{11}\right)$ receives a higher utility from this party than from voting for the coalition partner mentioned in the vignette or from choosing something else. This can be formalized in the following way:

$$
y=Y_{j_{1} j_{2}} \Leftrightarrow\left\{\begin{array}{cc}
U_{j_{1}}^{1}>U_{k_{1}}^{1} \text { for all } & k_{1} \neq j_{1} ; k_{1}, j_{1} \in J \\
U_{j_{2}}^{2}\left|y_{1}>U_{k_{2}}^{2}\right| y_{1} \text { for all } \quad k_{2} \neq j_{2} ; k_{2}, j_{2} \in J
\end{array} .\right.
$$

As usual, we assume that these utilities are random variables and therefore can be partitioned into a systematic

7. For example, if a respondent intends to vote for party $A$ in the standard as well as the vignette decision, the outcome would be $Y_{11}$. If she intended to vote for party $A$ in the standard and party $B$ in the vignette decision, the outcome would be denoted as $Y_{12}$, and if she intended to vote for party $A$ in the standard decision and did something else in the vignette decision, the outcome would be $Y_{13}$.

8. The idea to derive such a sequential choice model is strongly influenced by Signorino (2003). Our adaptation, however, is tailored to analyze sequential choice decision in survey experiments.

9. To simplify notation we drop the reference to voter $i$. 
Table 1. Conceptualization of a Sequential Choice Process with Nine Transition Probabilities

\begin{tabular}{llll} 
& $y_{2}=1$ & $y_{2}=2$ & $y_{2}=3$ \\
\hline & & & \\
$y_{1}=1$ & $\pi_{11}$ & $\pi_{12}$ & $\pi_{23}$ \\
$y_{1}=2$ & $\pi_{21}$ & $\pi_{22}$ & $\pi_{23}$ \\
$y_{1}=3$ & $\pi_{31}$ & $\pi_{32}$ & $\pi_{33}$ \\
\hline
\end{tabular}

component $\left(V_{j_{t}}^{t}\right)$, which we will parameterize to provide a test of our theory about how coalitions signals work, and a stochastic component $\left(\epsilon_{t j_{t}}\right)$. To keep the estimation problem tractable we assume independent, identically distributed (iid) errors following a type I extreme value distribution and parameterize the systematic component accordingly to account for the sources of the interdependence between both decisions. ${ }^{10}$ Given those assumptions, the transition probability of intending to vote $j_{1}$ in the standard decision and $j_{2}$ in the vignette decision can be derived as follows:

$$
\begin{aligned}
\pi_{j_{1} j_{2}}= & \operatorname{Pr}\left[e_{1 j_{1}}-e_{1 k_{1}}>V_{j_{1}}^{1}-V_{k_{1}}^{1}\right] \\
& \times \operatorname{Pr}\left[e_{2 j_{2}}-e_{2 k_{2}}>V_{j_{2}}^{2}-V_{k_{2}}^{2}\right] .
\end{aligned}
$$

Thus, the transition probability $\pi_{j_{1} j_{2}}$ is equal to the probability to vote $j_{1}$ in the standard decision multiplied by the probability to vote $j_{2}$ in the vignette decision, which can be estimated by the following product of two multinomial logit models that are jointly estimated:

$$
\pi_{j_{1} j_{2}}=\frac{e^{V_{i j_{1}}^{1}}}{e^{V_{i 1}^{1}}+e^{V_{i 2}^{1}}+e^{V_{i 3}^{1}}} \times \frac{e^{V_{i j_{2}}^{2}}}{e^{V_{i 1}^{2}}+e^{V_{i 2}^{2}}+e^{V_{i 3}^{2}}} .
$$

For identification purposes we set the utilities $V_{i j_{3}}^{1}$ and $V_{i j_{3}}^{2}$ equal to zero. Thus, the utility for the two parties are defined relatively to not intending to vote for any of the two parties mentioned in the coalition vignette.

After deriving a statistical model to analyze a given choice situation, the next step is to tailor the systematic component directly to our theory. It consist of three parts. First, we need

10. It is well-known that iid violations can be simply produced by misspecified systematic components (Signorino 2003). A preferable strategy in many applied settings is therefore to account for potential sources of iid violations in the specification of the systematic component rather than the stochastic component of the model (Dow and Endersby 2004; Glasgow 2001). This is what we do. Nevertheless, we also tried to relax the assumption of iid errors through parameterizing the variance-covariance matrix (which turns out to be a $9 \times 9$ matrix) and assuming multivariate normal distributed errors accordingly. Unfortunately, there is simply not enough information to reliably estimate at least some unconstrained parameters in the variancecovariance matrix. This is a well-known limitation in practical applications of such models. to parameterize the effect of party and coalition considerations to construct a valid theory test. Second, we account for the source of interdependence of the sequential choices by adding a dummy $y_{1 j}$ to the systematic component of the vignette decision indicating whether a respondent has already reported the same vote intention in the standard decision (i.e., $y_{1 j}=1$ if and only if $y_{1}=j$ ). ${ }^{11}$ Third, there might be other factors that explain a respondent's vote intention. We therefore add party-specific covariates and sociodemographic controls to the utility specification. Together, those three parts give us the following specification of the systematic component:

$$
\begin{aligned}
& V_{i j_{1}}^{1}=\beta_{1 j}+\lambda_{1}\left[\gamma_{1} P_{i j}+\left(1-\gamma_{1}\right) C_{i}\right]+\mathbf{Z}_{\mathbf{i j}} \boldsymbol{\delta}_{1}+\mathbf{X}_{\mathbf{i}} \psi_{\mathbf{j} 1}, \\
& V_{i j_{2}}^{2}=\beta_{2 j}+\lambda_{2}\left[\gamma_{2} P_{i j}+\left(1-\gamma_{2}\right) C_{i}\right]+\mathbf{Z}_{\mathbf{i j}} \boldsymbol{\delta}_{2}+\mathbf{X}_{\mathbf{i}} \psi_{\mathbf{j} 2}+\alpha y_{1 j} .
\end{aligned}
$$

We specify different parameters for the utility in the standard and vignette decision, ${ }^{12}$ including $\beta_{1 j}$ and $\beta_{2 j}$ as choice-specific constants $(j \in J)$. Coalition considerations $C_{i}$ and party considerations $P_{i j}$ are operationalized by like/ dislike rating scores that depict a voter's overall assessment of the parties and the coalition in the vignette. While $\lambda_{1}$ and $\lambda_{2}$ show the effect of party and coalition considerations in the standard and the vignette decision, $\gamma_{1}$ and $\gamma_{2}$ estimate the theoretically interesting mixing parameters. Allowing the mixing parameter to vary between the standard and the vignette decision permits us to directly test our theoretical expectation. If a coalition signal primes voters to rely more on coalition considerations, we should find a lower mixing parameter in the vignette than in the standard decision; that is, we expect $\gamma_{1}-\gamma_{2}>0$. The interdependence of the vote intention in the vignette decision from the vote intention in the standard decision is captured by $\alpha{ }^{13}$

11. For a similar strategy to represent previous states in a Markov transition as categorical variables, see Epstein et al. (2006). The cautious reader will realize that our assumption of iid errors is potentially violated. Again, as pointed out in note 10, it is a preferable strategy in applied settings to account for potential sources of iid violations directly (Dow and Endersby 2004; Glasgow 2001; Horowitz 1991) through the specification of the systematic component - in our case by including $y_{1 j}$-rather than the stochastic component of the model.

12. We opt for this flexible specification as we do not want to assume a priori that all effect parameters are the same in standard and vignette decisions. Given that we control for the vignette decision, whether the respondent has reported the same vote intention in the standard decision, the parameters might differ across the choice scenarios.

13. Note that the inclusion of such a dummy provides a rather conservative test of our theory because an increased reliance on coalition considerations might not necessarily lead to a change in behavior. The true effect of coalition signals could be greater than reported below. 


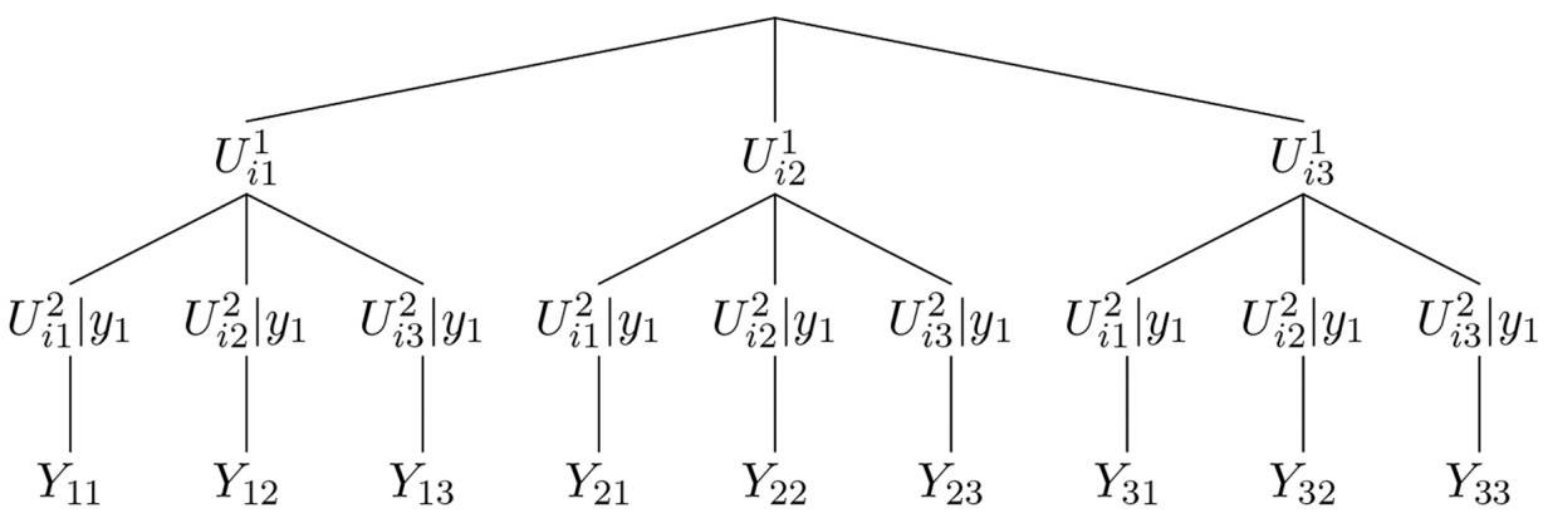

Figure 1. A sequential choice model

We further employ a set of controls that follows common practice in specifying vote choice models (see, e.g., Duch et al. 2010). The matrixes $\mathbf{Z}_{\mathbf{i j}}$ contain choice-specific covariates, controlling for the effect of an individual's party identification and the effect of the squared ideological distance to a party. The vectors $\boldsymbol{\delta}_{1}$ and $\boldsymbol{\delta}_{2}$ are the conditional effect parameters for these two controls for the standard and vignette decision. The matrix $\mathbf{X}_{\mathbf{i}}$ contains sociodemographic variables of the respondent (age, gender, education, religion, union membership, and income). ${ }^{14} \mathbf{y}_{\mathbf{j} 1}$ and $\mathbf{y}_{\mathbf{j} 2}$ are their effect parameters. To simplify notation, we collapse all parameters into a vector $\boldsymbol{\theta}$.

We estimate $\operatorname{pr}\left(\boldsymbol{\theta} \mid P_{i j}, C_{i}, \mathbf{Z}_{\mathbf{i j}}, \mathbf{X}_{\mathbf{i}}, y_{1 j}\right)$, the joint probability distribution of the parameters given the data, using Bayesian inference. Therefore, we iteratively sample from the posterior distribution, which is a product of the likelihood ${ }^{15}$ and the prior distribution $p(\Theta)$. In order to restrict the parameter space of the mixing parameters to the unit interval, we choose a uniform prior distribution on this interval, that is, $p\left(\gamma_{1}\right) \sim U(0,1)$ and $p\left(\gamma_{2}\right) \sim U(0,1)$, respectively. For all other parameters in the model we specify uninformative normal priors with mean zero and variance of 1,000 . Results are initially obtained by MCMC sampling running two chains for 20,000 iterations, discarding the first 18,000 iterations as burn-in. The model is implemented using JAGS (version 3.1.0). ${ }^{16}$

14. We refer the reader to the appendix for the way we operationalize those concepts.

15. The likelihood for our choice model is given by:

$$
L=\prod_{i=1 j_{1}}^{N} \prod_{1 j_{2}}^{3} \prod_{=1}^{3} \pi_{j_{1} j_{2}}^{\zeta_{j_{1}}},
$$

where $\zeta_{j_{1} j_{2}}=1$ if $y=Y_{j_{1} j_{2}}$ and 0 otherwise.

16. We checked model convergence using Heidelberger and Welch (1981) half-width test as well as the Gelman-Rubin diagnostic (Gelman and Rubin 1992). If all $\beta$ and $\delta$ passed the half-width test and if the upper bound of Gelman and Rubin's diagnostic was below 1.5, we considered the

\section{Results from the Austrian preelection study}

In this section we present the results from our surveyvignette experiment in context of the Austrian 2006 preelection study. First, we test whether respondents rely more on coalition considerations and less on party consideration for their vote choice when primed with coalition signal. Second, we assess the consequences of an increased importance of coalition considerations in an individual's decision-making calculus.

We provide four independent tests of our priming argument with the Austrian data. For each of the four vignettes we estimate one model using party and coalition considerations operationalized as respondents' like/dislike rating scores for parties and coalitions. ${ }^{17}$ Our quantities of interests to test our priming argument are the estimated mixing parameters. They indicate the relative weight voters attach to party and coalition considerations when forming their decision. ${ }^{18} \mathrm{We}$ present the estimated mixing parameters together with their Bayesian credible intervals across all four vignettes for the respective standard decision (black) as well as the vignette decision (gray) in the left panel of figure 2.

Overall, the results indicate strong support for our theory that coalition considerations become more important in a voter's decision calculus when primed with a coalition signal. Across all four tests we find the same expected pat-

model to have converged. We also conducted Geweke (1992) diagnostic tests. The appendix includes the test results as well as density and trace plots.

17. Unfortunately, there are no rating scores for a SPÖ-FPÖ coalition that is required to estimate the mixing parameters in our model. Thus, we have to slightly adjust the utility specification for this coalition vignette. Instead of a coalition rating, we employ the ratings of the respective coalition partner. In the appendix we show that while the respective mixing parameter will be measured on a different scale, we can still test whether it decreases in the vignette decision compared to the standard decision.

18. A complete overview over all estimated model parameters with Bayesian credible intervals can be found in table 7 in the appendix. 

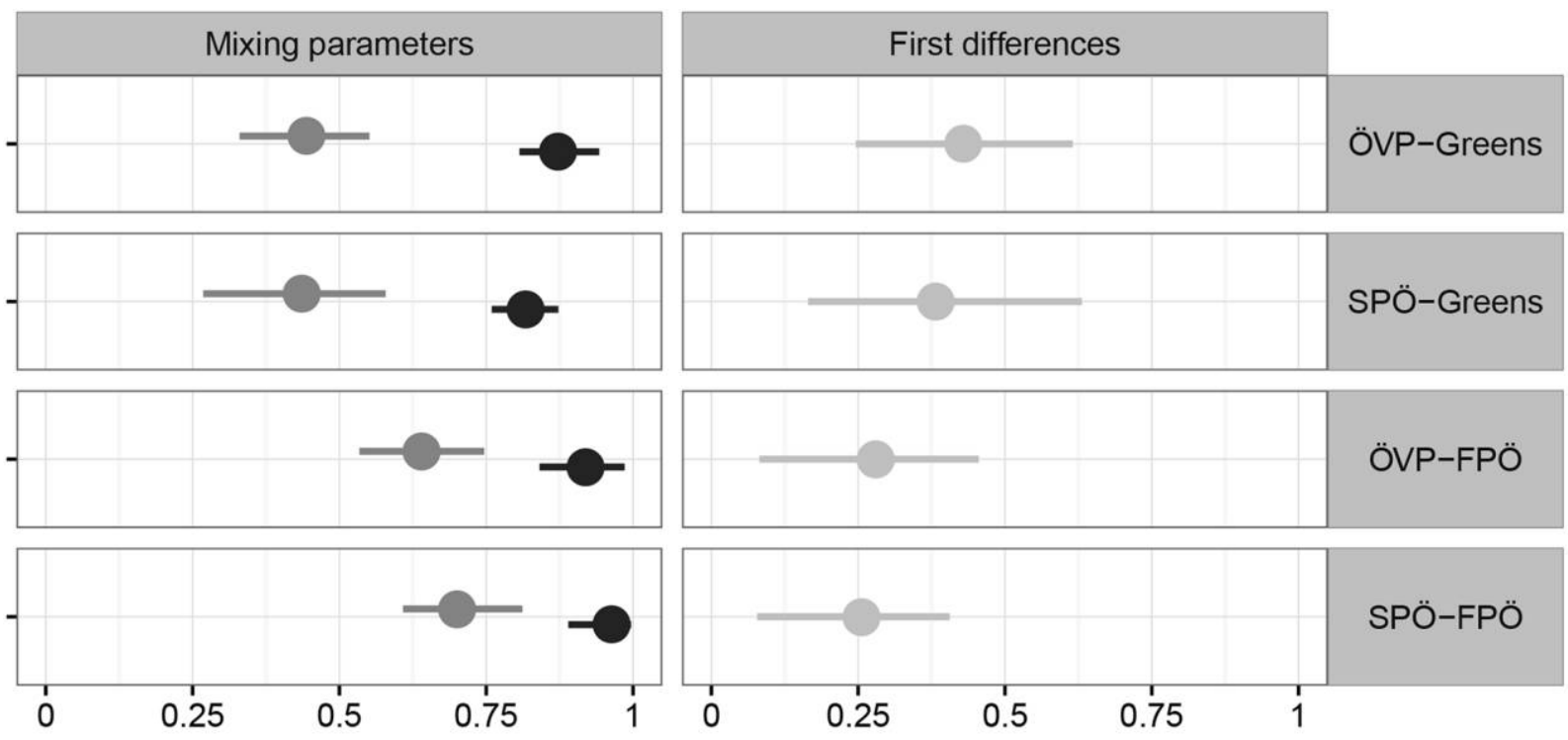

Standard

Vignette

First difference

Figure 2. Estimated mixing parameter in the Austrian preelection study. The left panel shows the estimated mixing parameter of the standard and the vignette decision. As expected, $\hat{\gamma}_{1}$ is always to the right of the respective estimated mixing parameter $\hat{\gamma}_{2}$ of the vignette decision. The right panel shows the first differences $\hat{\gamma}_{1}-\hat{\gamma}_{2}$ of the mixing parameters. Values to the right of 0 indicate that coalition considerations are weighted more heavily after being primed.

tern. The estimated mixing parameter is lower in the standard decision than in the vignette decision. Does this imply that the mixing parameter systematically decreases as expected when predicting the vote choice of the same respondent across both decisions? In order to answer this question and to provide a hard test for our theory, we need to show that the first difference between the two mixing parameters is positive, that is, $\hat{\gamma}_{1}-\hat{\gamma}_{2}>0$.

Our Bayesian model setup allows us to directly sample the first differences between the mixing parameter in the standard decision and the mixing parameter for the vignette decision from the posterior distribution. This tells us to what degree an average respondent weighs coalition considerations more heavily in the vignette decision as compared to the standard decision.

The right panel of figure 2 plots the first differences of the mixing parameters for all four coalition signals. It shows that the respective 95\% Bayesian credible intervals are to the right of 0 for each coalition signal. The simulations of the first differences imply that respondents systematically rely more on coalition considerations and at the same time less on party considerations when primed with a coalition signal. The lesson that can be learned so far is that coalition signals change the relative weights of party and coalition considerations in a voter's utility function. We find that coalition considerations for the coalition in each signal become more important relative to party considerations.
What are potential consequences of an increased importance of coalition considerations in an individual's decisionmaking calculus? If coalition signals are able to change the importance voters attach to the same yardsticks that determine their vote choice, then we should observe potential changes in their voting behavior when primed with a coalition signal. This does not necessarily imply that all voters change their vote intention. If someone is already very likely to vote for a certain party, then coalition signals might still increase the importance of coalition considerations but not lead to a different vote decision.

One way to asses this observable implication of our theory is to systematically compare how likely our model predicts respondents to vote for a particular party with and without being primed by a particular coalition signal. Given that we now know what coalitions signals do, we should find that an increased reliance on particular coalition considerations might lead voters to reconsider their standing vote decision. This should be the case for supporters of a coalition party who do not like this coalition. When primed by a coalition signal in the vignette decision the predicted probabilities to vote for one of those parties should decrease the more this coalition is disliked. We do not expect such a relationship between coalition preferences and vote intention when coalition considerations are not primed, that is, in the standard decision.

Figure 3 shows exactly this. We plot the predicted probabilities of voting for the Greens based on our model for the 


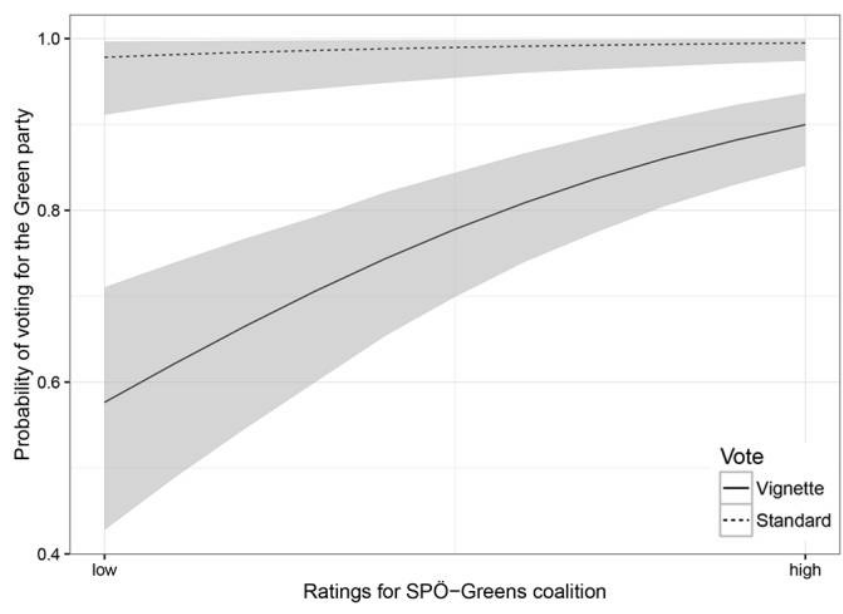

Figure 3. Probability to vote for Greens in standard vs. SPÖ-Green vignette decision by different levels of ratings for SPÖ-Green coalition.

standard decision (dashed line) and compare those to the ones of the vignette decision (solid line) that primed a coalition of SPÖ and Greens. The predicted probabilities are sampled from the posterior distribution for an otherwise typical respondent that rates the Green party with the highest possible score and the SPÖ with an average score. We further assume that our respondent does not identify with any of the two parties, and intends to vote for the Greens in the standard decision. The figure shows how predicted probabilities in both decisions vary with different coalition ratings. For the vignette decision, predicted probabilities increase from $0.63([0.47,0.75])$ for the lowest coalition rating to 0.92 $([0.87,0.95])$ for the highest rating score, while the effect of coalition ratings is negligible without priming this coalition in the standard decision. Predicted probabilities only increase from 0.98 to 0.99 with overlapping credible intervals ([0.93, 0.99] and [0.98, 0.99]). Thus, Green voters are more likely to change their vote intention if they dislike the primed coalition in the coalition signal. ${ }^{19}$

To sum up, these results illustrate that coalition signals prime coalition considerations. Coalition signals increase the importance of coalition considerations in relation to party considerations when voters make up their mind who to vote for on election day. Furthermore, the increased importance of coalition considerations through priming can have important behavioral consequences. The proclivity to vote for one of those parties in the coalition signal decreases the more this coalition is disliked. This might cause voters to change their voting behavior. Do these findings about the impact of coalition signals depend on the particular party system or

19. We observe a similar pattern for other coalition signals, see the appendix, figure 1 . electoral context for which we drafted those vignettes? This is fortunately not the case as we show in the next section.

\section{Results from the German Longitudinal Election Study}

In order to show that our results travel across time and countries, we present the results of four more comparable survey vignettes representing particular coalition signals that were implemented in the 2009 GLES (German Longitudinal Election Study). While two vignettes prime coalitions between two parties similar to the Austrian case, the two remaining vignettes prime relevant three-party coalitions. ${ }^{20}$ As before, we estimate our model for each of the four vignettes in the GLES separately.

For all four models we estimate the mixing parameters to be lower in the vignette decision than in the standard decision. ${ }^{21}$ But how likely is it that the mixing parameter of the vignette decision is lower than the mixing parameter of the standard decision? In order to answer this question we inspect - as before in the Austrian case - the respective first differences of the mixing parameters. We provide the mean together with their $95 \%$ credible interval for each of the first differences in left panel of figure 4.

The results for the coalition signals representing both twoparty coalitions provide strong support for our theory. The figure clearly shows that the credible intervals do not cross the reference line at zero. In fact, although implemented in a different country at a different time within idiosyncratically different election campaigns, and using slightly different designs and wordings, the estimated size of the first differences between the mixing parameters of each model are comparable for all two-party coalitions in Austria and Germany.

The results for the coalition signals representing the threeparty coalitions look similar although the estimated size of the respective first differences is generally smaller. Moreover, their credible intervals intersect with the reference line. From our posterior draws, however, we can calculate the likelihood that the differences of the estimated mixing parameters fit our expectation. Looking at the posterior draws of the respective first differences, we find that with a probability of 95.5\% respondents weigh their coalition preferences higher when primed with an appropriate coalition signal compared

20. In order to make use of vignettes of three-party coalitions, we straightforwardly extend our model to $4 \times 4$ choices to account for a larger choice set. Each respondent could report an intention to vote for one of the three parties in such a coalition or, as before, do something else. For a more detailed description of the model we refer to the appendix of the article.

21. We present mean and 95\% Bayesian credible intervals of the parameters' posterior distributions of each model in table 15 in the appendix. 

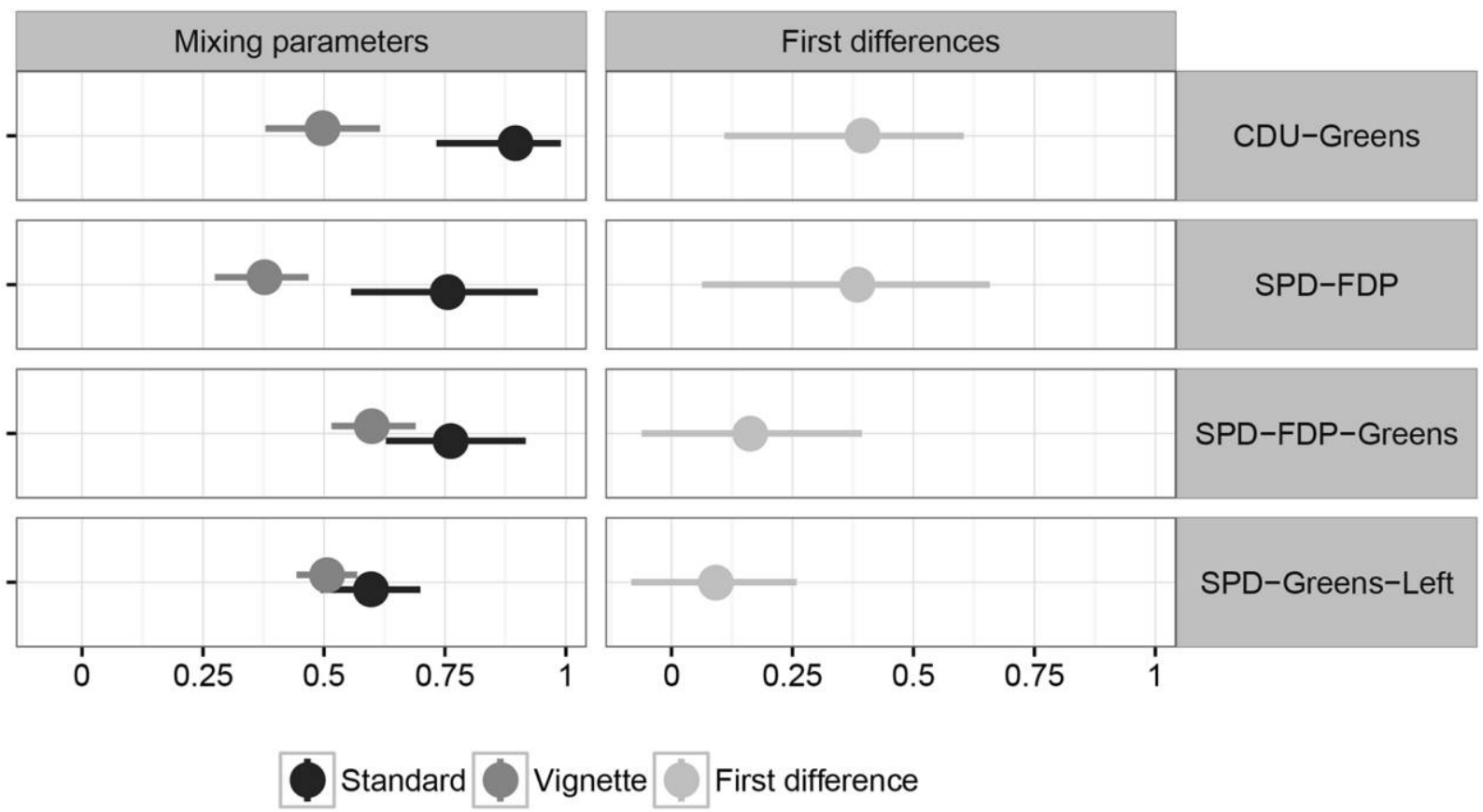

Figure 4. Estimated mixing parameter in the German Longitudinal Election Study. The left panel shows the estimated mixing parameter of the standard and the vignette decision. As expected, $\hat{\gamma}_{1}$ is always to the right of the respective estimated mixing parameter $\hat{\gamma}_{2}$ of the vignette decision. The right panel shows the first differences $\hat{\gamma}_{1}-\hat{\gamma}_{2}$ of the mixing parameters. Values to the right of 0 indicate that coalition considerations are weighted more heavily after being primed.

to the standard decision. This makes us fairly confident that there is a systematic difference between the two decisions as expected. Even when primed with an SPD-Greens-Left coalition signal we are at least $89.7 \%$ certain that respondents rely more on their respective coalition preferences rather than their party preferences. ${ }^{22}$ At the same time, one has to acknowledge the increased complexity of the estimation problem. Although the extension of our research strategy to include three-party coalitions is conceptually and theoretically straightforward, this extension comes at a high cost. The number of outcomes increases from 9 in the two-party coalition case to 16 in a three-coalition case. Some of these outcomes are rarely observed, which makes it harder to reliably estimate the theoretically interesting mixing parameters of our model.

Overall, the results from Germany are converging with the results from Austria. Together they provide consistent evidence for our priming argument and do not seem to depend on either a particular election context or on a specific party system. ${ }^{23}$ Coalition signals prime voters to rely more

22. The respective probabilities for the two-party coalition signals indicate that almost the entire support of the distribution is on the unit interval.

23. We further checked the robustness of our results against unobserved confounders. Section G, in the appendix of this article, reports that the results hold across five randomly constructed three-quarter subsets of the respective data sets. on coalition preferences when forming their decision. Coalition signals increase the importance of coalition considerations and, at the same time, decrease the importance of party considerations when voters make up their mind. This can lead voters to reconsider their standing vote choice decision and change their voting behavior.

Finally, there is also converging evidence across our Austrian and German results that coalition signals not only do not confuse voters but rather seem to facilitate better decisions by priming relevant considerations. When comparing descriptively the transition matrices between standard and vignette decision we find that no matter which of the vignettes is administered, there are more German and Austrian nonvoters who are mobilized and report a vote intention after being exposed to vignettes than voters who are demobilized. ${ }^{24}$ Thus, there is a positive net-effect indicating that coalition signals consistently have the potential to increase turnout and reduce voter confusion.

\section{CONCLUSION}

Coalition signals are an important preelectoral strategy for parties during election campaigns in multiparty democra-

24. We refer the interested reader to tables 2 and 14 in appendixes A and $\mathrm{C}$, respectively. For further supporting evidence, see also Bytzek et al. (2011, 402). 
cies. Such coalition signals seem to remind voters about the outcome of the election and the coalition bargaining process. In our survey experiments, they increase our respondents' reliance on coalition considerations at the expense of party considerations when formulating their vote choice decision. Coalition signals can even lead voters to systematically reconsider their standing vote choice decision. Given the converging evidence based on data from survey vignettes representing coalition signals in two countries, Austria and Germany, our results do not seem to depend on a particular election context, party system, or a specific operationalization and wording of the vignettes.

At first sight, coalition signals seem to distract voters from determining which party they prefer most. If this were true, it would reflect poorly on popular conceptions of democratic representation. However, our results rather suggest the opposite. Coalition signals not only do not confuse voters but rather facilitate better decisions by priming relevant considerations. They remind voters that a coalition of parties and not a single party alone will form the government after the election. They also help voters to figure out which party they should support to vote their most preferred coalition into office. This also nicely complements previous accounts from Belgium (Gschwend and Hooghe 2008) and Poland (Kaminski 2001, 302), indicating that voters do not blindly follow those signals. Thus, coalition signals can offer valuable information to hold the government accountable and to identify potential future governments, particularly in an environment that is typically characterized as providing voters only with low levels of accountability.

Moreover, it is important to keep in mind that our analysis only identifies the effect of coalition signals if they yield a change in party choice when comparing the vote intentions before and after exposure to coalition vignettes. The true effect of coalition signals could be greater than reported here if, for example, reinforcement effects are taken into account. A small party supporter might become even more inclined to vote for her party after hearing that the party is willing to join a coalition with a larger party - assuming that this voter strongly approves such a coalition.

While we provide evidence in support of priming as a mechanism how coalition signals work, coalition signals could instead change voters' expectations about which coalitions are likely to emerge in the postelection bargaining period. Thus, rather than priming specific coalition considerations, coalition signals could primarily reduce the number of theoretically possible coalitions. According to such an alternative expectation-formation account they help citizens to form clearer expectations about the government formation process after the election. A positive coalition signal would indicate that, conditional on getting a majority of seats in the legislature, the parties in the signal will actually form the government. But how to distinguish the expectation-formation account from a pure priming story we propagate here? There is no priming effect without changing voters' expectations about which coalitions are likely to expect after the electionand vice versa. Every change in voters' expectations about parties' willingness to cooperate should increase the importance of coalition considerations in a voter's decision-making process. Whether a positive coalition signal conditions the postelectoral expectations or, as we would argue, increases the importance of consideration about a specific coalition, both mechanisms yield the same observable implication: Coalition vignettes should positively increase the impact of the respective coalition considerations in a voter's decision-making process. Future research should try to design a study that is able to disentangle the priming from the expectation-formation account.

While we have identified a plausible mechanism how coalition signals can work and influence an individual's decisionmaking process, we do not have direct evidence of the precise cognitive processes that are triggered when citizens are primed to think about coalitions. For example, this process could be highly affective or purely policy-driven. Do citizens who are primed with coalition signals think about the expected policy position of the potential coalition governments and finally decide if it is still worth voting for one of the parties in that signal, or do they rather rely on likability considerations? The survey data we have is not ideally suited to disentangle the cognitive processes that underlie electoral decision making. Nevertheless, our results highlight that the electoral competition between parties can organize the psychological processes voters employ to make their decisions. Following this line of thought, we might expect that coalition signals that also mention concrete programmatic platforms or policies will initiate a policy-driven reaction instead of an affective one.

To further put our conclusions in context, we need to point out that all coalition signals used here were reasonable but clearly hypothetical, making any prediction of real world impact highly tentative. It is more or less impossible to manipulate the actual coalition signals of real parties, especially within a nationally representative survey. Our goal was to test theoretical expectations about the causal mechanism outside the lab rather then estimating their real-world consequences in an actual campaign. Further research on the nature and role of coalition signals needs to pay more attention to the heterogeneity of such effects caused by positive and negative coalition signals or by different sources of such signals. 
Our study has very clear theoretical and practical implications. Theoretically, coalition signals require more attention. There seems to be a consensus among the few studies that explored the effects of coalition signals that voters appear to take them systematically into account when making a decision. Theories of voting behavior must account for such preelectoral party strategies as contextual features. In order to understand the electoral impact of such strategies, scholars need to understand when and why parties send out which type of coalition signal. Our study therefore contributes to an emerging literature that sees behavioral and institutional explanations of party competition as allies rather than rivals (see, e.g., Sniderman and Levendusky 2007).

There are also rather practical implications for our understanding of party competition. Our results suggest that coalition signals can have real electoral costs and benefits for parties. They require careful thought about the consequences, whether a signal will benefit or hurt a party, or even affect a third party not included in the signal. Parties want to make sure that they have a net benefit from the gains and losses when sending out specific signals. Thus, our theory allows to identify conditions under which parties should have a strong incentive to send out coalition signals during election campaigns. For instance, if a coalition is more popular than the party itself, or if the coalition position is closer to the median voter, rational parties should send a respective coalition signal. Future research can build on this finding to better understand the conditions under which parties are especially likely to form preelectoral coalitions.

Finally, our strategy to estimate the relative importance of competing mechanisms across different contexts can be applied not just to parties and coalitions but to other decisionrelevant considerations as well. The literature on information environments (Jerit, Barabas, and Bolsen 2006; Kuklinski et al. 2001) could use designs similar to our coalition vignettes to show under what conditions certain information environments affect the quality of decisions. A similar model to ours could be employed in the areas of political behavior and political psychology to test the importance of primed information, for instance for certain policies, and their behavioral consequences. Even a comparative approach is possible, for example, by studying the behavioral responses in different contexts by comparing the importance of the EU-integration dimension for vote choice in EU and national elections. We have shown that coalition signals create a new context that influences decision making by emphasizing different decision criteria. Thus, similar designs to the one used here should help scholars to test observable implications of contrasting mechanisms that drive political decisions in different contexts.

\section{ACKNOWLEDGMENTS}

For helpful comments, we are grateful to Sean Carey, Indridi Indridason, Orit Kedar, Franz Pappi, Daniel Stegmueller, Markus Wagner, and Steffen Zittlau. Parts of this research have been conducted while Gschwend was Visiting Professor at the Department of Social Science, Utrecht University, and at the Institute for Advanced Studies, Vienna. Lukas Stoetzer conducted most of the research at the University of Mannheim. Both would like to thank those institutions for their hospitality, their stimulating research environment, and all seminar participants for helpful comments.

\section{REFERENCES}

Aldrich, John H., André Blais, Indridi H. Indridason, and Renan Levine. 2004. "Coalition Considerations and the Vote." In Asher Arian and Michal Shamir, eds., The Elections in Israel, 2003. New Brunswick, NJ: Transaction, 180-211.

Allern, Elin Haugsgjerd, and Nicholas Aylott. 2009. "Overcoming the Fear of Commitment: Pre-Electoral Coalitions in Norway and Sweden.” Acta Politica 44 (3): 259-85.

Armstrong, David A., and Raymond M. Duch. 2010. "Why Can Voters Anticipate Post-Election Coalition Formation Likelihoods?" Electoral Studies 29 (3): 308-15.

Bargsted, Matias A., and Orit Kedar. 2009. "Coalition-Targeted Duvergerian Voting: How Expectations Affect Voter Choice under Proportional Representation.” American Journal of Political Science 53 (2): 307-23.

Bartels, Larry M. 2006. "Priming and Persuasion in Presidential Campaigns." In Richard Johnston and Henry E. Brady, eds., Capturing Campaign Effects. Ann Arbor: University of Michigan Press, 78-112.

Blais, André, John H. Aldrich, Indridi H. Indridason, and Renan Levine. 2006. "Do Voters Vote for Government Coalitions? Testing Downs' Pessimistic Conclusion." Party Politics 12 (6): 691-705.

Blais, André, and Indridi H. Indridason. 2008. "Making Candidates Count: The Logic of Electoral Alliances in Two-Round Legislative Elections." Journal of Politics 69 (1): 193-205.

Bowler, Shaun, Jeffrey A. Karp, and Todd Donovan. 2010. "Strategic Coalition Voting: Evidence from New Zealand.” Electoral Studies 29 (3): 350-57.

Bytzek, Evelyn, Thomas Gschwend, Sascha Huber, Eric Linhart, and Michael F. Meffert. 2011. "Koalitionssignale und ihre Wirkungen auf Wahlentscheidungen." Politische Vierteljahresschrift 45:393-418.

Clark, William R., Matt Golder, and Sona N. Golder. 2012. Principles of Comparative Politics. 2nd ed. Washington, DC: CQ Press.

Debus, Marc. 2009. "Pre-Electoral Commitments and Government Formation." Public Choice 138 (1-2): 45-64.

Diggle, Peter, Patrick Heagerty, Kung-Yee Liang, and Scott Zeger. 2002. Analysis of Longitudinal Data. 2nd ed. New York: Oxford University Press.

Dow, Jay K., and James W. Endersby. 2004. "Multinomial Probit and Multinomial Logit: A Comparison of Choice Models for Voting Research." Electoral Studies 23 (1): 107-22.

Duch, Raymond M., Jeff May, and David A. Armstrong II. 2010. "Coalition-Directed Voting in Multiparty Democracies." American Political Science Review 104 (4): 698-719.

Epstein, David, Robert H. Bates, Jack Goldstone, Ida Kristensen, and Sharyn O’Halloran. 2006. “Democratic Transitions.” American Journal of Polictical Science 50 (3): 551-69.

Fredén, Annika. 2014. “Threshold Insurance Voting in PR Systems: A Study of Voters' Strategic Behavior in the 2010 Swedish General Election." Journal of Elections, Public Opinion and Parties 24 (4): 1-20. 
Gelman, Andrew, and Donald B. Rubin. 1992. "Inference from Iterative Simulation Using Multiple Sequences." Statistical Science 7 (4): 45772.

Geweke, John. 1992. "Evaluating the Accuracy of Sampling-Based Approaches to the Calculation of Posterior Moments." In José M. Bernardo, James O. Berger, A. Philip Dawid, and Adrian F. M. Smith, eds., Bayesian Statistics 4. Oxford: Oxford University Press, 169-93.

Glasgow, Garrett. 2001. "Mixed Logit Models for Multiparty Elections." Political Analysis 9 (2): 116-36.

Golder, Sona Nadenichek. 2005. "Pre-Electoral Coalitions in Comparative Perspective: A Test of Existing Hypotheses." Electoral Studies 24 (4): 643-63.

Golder, Sona Nadenichek. 2006. "Pre-Electoral Coalition Formation in Parliamentary Democracies." British Journal of Political Science 36 (2): 193-212.

Gschwend, Thomas. 2007a. "Institutional Incentives for Strategic Voting and Party System Change in Portugal." Portuguese Journal of Social Science 6 (1): $15-31$.

Gschwend, Thomas. 2007b. "Ticket-Splitting and Strategic Voting under Mixed Electoral Rules: Evidence from Germany." European Journal of Political Research 46 (1): 1-23.

Gschwend, Thomas, and Marc Hooghe. 2008. "Should I Stay or Should I Go? An Experimental Study on Voter Responses to Pre-Electoral Coalitions." European Journal of Political Research 47 (5): 556-77.

Gschwend, Thomas, Lukas Stoetzer, and Steffen Zittlau. 2016. "What Drives Rental Votes? How Coalitions Signals Facilitate Strategic Coalition Voting." Electoral Studies 44:293-306.

Heidelberger, Philip, and Peter D. Welch. 1981. "A Spectral Method for Confidence Interval Generation and Run Length Control in Simulations." Communications of the ACM 24 (4): 233-34.

Herrmann, Michael. 2014. "Polls, Coalitions and Strategic Voting under Proportional Representation." Lournal of Theoretical Politics 26 (3): $442-67$.

Hillygus, D. Sunshine. 2007. "The Dynamics of Voter Decision Making among Minor-Party Supporters: The 2000 Presidential Election in the United States." British Journal of Political Science 37 (2): 225-44.

Hobolt, Sara B., and Jeffrey A. Karp. 2010. "Voters and Coalition Governments." Electoral Studies 29 (3): 299-307.

Horowitz, Joel L. 1991. "Reconsidering the Multinomial Probit Model." Transportation Research Part B: Methodological 25 (6): 433-38.

Irwin, Galen A., and Joop J. M. Van Holsteyn. 2008. "What Are They Waiting For? Strategic Information for Late Deciding Voters." International Journal of Public Opinion Research 20 (4): 483-93.

Irwin, Galen A., and Joop J. M. Van Holsteyn. 2012. "Strategic Electoral Considerations under Proportional Representation.” Electoral Studies 31 (1): 184-91.
Jerit, Jennifer, Jason Barabas, and Toby Bolsen. 2006. "Citizens, Knowledge, and the Information Environment." American Journal of Political Science 50 (2): 266-82.

Kaminski, Marek M. 2001. "Coalitional Stability of Systems: Evidence from Poland." American Journal of Political Science 45 (2): 294-312.

Kedar, Orit. 2011. "Voter Choice and Parliamentary Politics: An Emerging Research Agenda." British Journal of Political Science 42 (3): 537-53.

Kuklinski, James H., Paul J. Quirk, Jennifer Jerit, and Robert F. Rich. 2001. "The Political Environment and Citizen Competence." American Journal of Political Science 45 (2): 410.

Lewis-Beck, Michael S., and Andrew Skalaban. 1989. "Citizen Forecasting: Can Voters See into the Future?” British Journal of Political Science 19 (1): 146-53.

Linhart, Eric. 2009. "A Rational Calculus of Voting Considering Coalition Signals: The 2005 German Bundestag Election as an Example." World Political Science Review 5 (1): 461-84.

Martin, Lanny W., and Randolph T. Stevenson. 2001. "Government Formation in Parliamentary Democracies." American Journal of Political Science 45 (1): 33-50.

Martin, Lanny W., and Randolph T. Stevenson. 2010. "The Conditional Impact of Incumbency on Government Formation." American Political Science Review 104 (3): 503-18.

Meffert, Michael F., and Thomas Gschwend. 2010. "Strategic Coalition Voting: Evidence from Austria." Electoral Studies 29 (3): 339-49.

Meffert, Michael F., and Thomas Gschwend. 2011. "Polls, Coalition Signals and Strategic Voting: An Experimental Investigation of Perceptions and Effects." European Journal of Political Research 50 (5): 636-67.

Meffert, Michael F., and Thomas Gschwend. 2012. "Experimental Triangulation of Coalition Signals: Varying Designs, Converging Results." In Bernhard Kittel, Wolfgang J. Luhan, and Rebecca B. Morton, eds., Experimental Political Science. Principles and Practices. New York: Palgrave Macmillan, 140-60.

Meffert, Michael F., Sascha Huber, Thomas Gschwend, and Franz Urban Pappi. 2011. "More than Wishful Thinking: Causes and Consequences of Voters' Electoral Expectations about Parties and Coalitions." Electoral Studies 30 (4): 804-15.

Powell, G. Bingham. 2000. Elections as Instruments of Democracy: Majoritarian and Proportional Visions. New Haven, CT: Yale University Press.

Signorino, Curtis S. 2003. "Structure and Uncertainty in Discrete Choice Models." Political Analysis 11 (4): 316-44.

Sniderman, Paul M., and Matthew S. Levendusky. 2007. "An Institutional Theory of Political Choice." In Russell J. Dalton and HansDieter Klingemann, eds., The Oxford Handbook of Political Behavior. Oxford: Oxford University Press, 437-57.

Strom, Kaare. 1990. Minority Government and Majority Rule. Cambridge: Cambridge University Press. 